International Research Journal of Public and Environmental Health Vol.9 (1),pp. 10-15, February, 2022

Available online at https://www.journalissues.org/IRJPEH/

https://doi.org/10.15739/irjpeh.22.002

Copyright (C) 2022 Author(s) retain the copyright of this article

ISSN 2360-8803

\title{
Cervical cancer elimination: Readiness to achieve interim targets in Sri Lanka 2021 - 2030
}

Upuli Perera $^{1}$, Thanuja

Wickramatunga ${ }^{2}$,

Kalpanie Wijewardane ${ }^{2}$, Saddharma Weerakoon ${ }^{3}$ and

\section{Janaki Vidanapathirana ${ }^{4}$}

${ }^{1}$ Consultant Community

Physician, National Cancer

Control Programme, Ministry of health, Sri Lanka.

2Medical Officer, National Cancer

Control Programme Ministry of health, Sri Lanka.

${ }^{3}$ Registrar in Community

Medicine, National Cancer

Control Programme, Ministry of health, Sri Lanka.

${ }^{4}$ Director, National Cancer

Control Programme Ministry of health, Sri Lanka.

*Corresponding Author Email : tauapp100@gmail.com

Tel.:: +94718043649
Cervical cancer is highly preventable and curable cancer. In 2020, World Health Organization has declared a global strategy to eliminate cervical cancer as a public health problem. The study's goal is to create a National Strategic Plan (NSP) to achieve the interim goal of eliminating cervical cancer as a public health problem. There was an identification of the vision, mission, goal, strategic objectives, and strategic directions. The National Cancer Control Programme developed the initial draught of the NSP based on a set of guiding principles, national and international policies, and the global strategy. Feedback was obtained via a series of hybrid meetings from stakeholders and the content was finalized. With the goal to reach interim targets for elimination of cervical cancer by 2030, the NSP was developed as a guiding document for Sri Lanka's response to elimination of cervical cancer. This document has laid down seven strategic objectives, several strategic directions and major activities to achieve 90-70-90 targets of elimination. It ensured the strengthening of existing strategies for cervical cancer care and identified the new strategies. The NSP was developed to reach the interim targets of cervical cancer elimination in Sri Lanka by 2030.

Keywords: Cervical cancer, interim targets, elimination, strategic plan, Sri Lanka

\section{INTRODUCTION}

Cervical cancer is preventable and curable when detected early and treated effectively (World Health Organization, 2021a). The primary cause of cervical cancer is the persistent infection with a high risk or oncogenic type human papilloma virus (HPV) infection (World Health Organization, 2021a). HPV types 16, 18 are the most oncogenic and together are responsible for approximately $70 \%$ of cervical cancer world-wide (de Martel et al., 2017). Most HPV infections are transient and resolve spontaneously. Only few infections are persistent and only very few progresses to pre-cancerous lesions and invasive cancer. It takes 10-15 years for a precancerous lesion to develop into an invasive cancer (de Martel et al., 2017). Other risk factors causing this cancer are classified as cofactors favoring acquisition of HPV infection and co-factors favoring persistence of HPV infection (National Cancer Control Programme, 2020). Commencement of sexual activity at an early stage, multiple sexual partners, partner having multiple sexual partners, poor socioeconomic status, poor hygiene, immunosuppression, high parity, and young 
age at first childbirth are the co factors favouring acquisition of HPV infection. HPV type - its oncogenicity, immune status, co-infection with other Sexually Transmitted Infections, high parity \& young age at first birth, tobacco smoking, poor socioeconomic status and prolonged use of Oral Contraceptive Pills are the factors favouring persistence of HPV infection (National Cancer Control Programme, 2020).

There is a known definitive cause and available methods to prevent high-risk HPV types in cervical cancer. Further the disease progression from initial infection to invasive cervical cancer is slow. Above reasons make this cancer a highly preventable one if it can be detected early. (World Health Organization, 2020). Over the years, vaccination against HPV, screening and treatment of pre-cancer, early detection, and prompt treatment of early invasive cancers and palliative care are global public health strategies. Those strategies have effectivity proven to address cervical cancer in relation to primary, secondary and tertiary preventive strategies. In 2020, World Health Organization (WHO) recognized the need to eliminate cervical cancer as a public health problem and declared a global strategy (World Health Organization, 2020).

Cervical cancer has become the fourth most common cancer among women and the fourth leading cause of cancer related deaths in women in the world (World Health Organization, 2021a). Globally, an estimated 604,127 women diagnosed with cervical cancer $(3.1 \%)$ and around 341,831 women died due to cervical cancer (3.3\%) in 2020. Cervical cancer is one of the diseases which reveal global inequities (World Health Organization, 2021a). In 2020, WHO recognized the need to eliminate cervical cancer as a public health problem, with a threshold of 4 per 100,000 women-years at the end of the century. $90 \%$ of girls fully vaccinated with the HPV vaccine by 15 years of age; $70 \%$ of women screened using a high-performance test by 35 years of age and again by 45 years of age; $90 \%$ of women identified with cervical disease are treated are the set interim targets to be achieved by 2030. Elimination of cervical cancer will contribute to the achievement of several Sustainable Development Goals (SDG) including goal 1, 3, 5 and 10. Ultimately, reaching the interim targets lead to the pathway to achieve the SDGs globally (United Nations, 2015).

Cervical cancer was ranked fourth position from 2015 among all cancers in females, while in 2019 it comprised of $6 \%(\mathrm{n}=1114)$ of all cancers in females in Sri Lanka (National Cancer Control Programme, 2021a). The crude incidence rate (CR) showed a slight increase in trend from 2005 to 2019 where it fluctuated between 7 to 10 per 100,000 female population. The Average Annual Percent Change of CR of cervical cancer from 2005 to 2019 was increased by $1.7 \%$ annually (National Cancer Control Programme, 2021a). Even with the lack of staging information in Sri Lankan National Cancer Registry data, late diagnosis is evident. $52.7 \%, 38.7 \%, 39.1 \%$ and $49.6 \%$ have been diagnosed in stage III and IV in year 2016, 2017, 2018 and 2019 respectively (National Cancer Control
Programme, 2021a).

As the focal point for cancer prevention and control, National Cancer Control Programme (NCCP) has implemented various strategies in relation to cervical cancer. Prevention of risk factors for cervical cancer is integrated to several health and non-health programmes. Island-wide quadrivalent HPV vaccination has been introduced into the Expanded Programme of Immunization in Sri Lanka in 2017. Two doses of the vaccine are given to girls aged 10 and 11 with a minimum interval of six months. Currently the programme is reaching the set targets and demand is already created in the country to maintain high coverage (Epidemiology Unit, 2020). Pap smear screening among 35- and 45-year age cohorts are currently carried out through the island-wide well woman progrmme in Family Health Bureau (FHB) at each Medical Officer of Health (MOH) area under Well Woman Programme (WWP). 35 and 45 age cohort attendance to WWC in 2019 is 59.1\% and $25.5 \%$ respectively (Family Health Bureau, 2019). According to the WHO recommendation, Sri Lanka has initiated an island-wide transition of HPV-DNA testing and a pilot study was completed (Perera et al., 2021).

Establishment of Cancer Early Detection Centers, Centers of Excellence for cancer diagnosis and treatment and colposcopy centers are some of the initiatives taken to strengthen the cervical cancer care in Sri Lanka (National Cancer Control Programme, 2021b). Treatment services for cervical cancer including precancerous lesions and invasive cancer are available at the Apeksha Hospital, Maharagama, which is the premier center for treatment of adults and children with cancer, and 24 other cancer treatment centres distributed across the country. Current treatment modalities offered include surgery, chemotherapy and radiotherapy including brachytherapy. Brachytherapy facilities are limited at the moment and initiation has taken to expand the services (National Cancer Control Programme, 2021b).

Lack of knowledge coupled with stigma, myths and misconceptions are being identified as constraints in reaching the expected targets of the cervical cancer care in the country (de Silva et al., 2019). High cost for screening methods such as HPV DNA tests, lack of mainstreamed referral and back referral services and lack of patient management information services are also identified as challenges (National Cancer Control Programme, 2021c). It has been identified that there are gaps in reaching all 3 global set targets in Sri Lanka. The Development of this NSP therefore identified as an essential requirement to reach these interim targets by 2030 . This decision was taken at a wide stakeholder meeting chaired by the Director General of Health Services. This NSP leads the pathway to ensure and strengthen the already existing strategies while to develop and implement the new strategies to fulfill the gaps. Ultimately, this will lead to assess the whole programme ensuring the set targets will be achieved by 2030 (National Cancer Control Programme, 2021c).

Aim of the study is to develop the NSP to reach the interim targets of the elimination of cervical cancer as a 
public health problem by 2030 .

\section{MATERIALS AND METHODS}

The draft of the NSP was developed based on the several national and international policies, strategies and guidelines. Some of them are the Global strategy to accelerate the elimination of cervical cancer as a public health problem (World Health Organization, 2020), Regional implementation framework for elimination of cervical cancer as a public health problem 2021-2030 (World Health Organization, 2021b) and National Strategic Plan on Prevention and Control of Cancer in Sri Lanka (2020-2024) (National Cancer Control Programme, 2021b). Further, the local context and Sri Lankan evidence were considered. It was finalized by number of virtual meetings with stakeholders including FHB, Epidemiology Unit, NonCommunicable Diseases unit, local WHO partners, members of the National Advisory Committee on Cancer Prevention and Control, Technical Advisory Committee of the cancer prevention and control \& Diagnosis and Treatment, relevant professional colleges, Non-Governmental Organization (NGO) partners and Community Based organizations.

A sufficient time was given for review and feedback. Further, several communications were done via emails /telephone calls for those who were not responding on time. Later, clarification was done based on their feedback where necessary, and updated drafts were circulated three times. In addition, NCCP conducted several virtual meetings with the above stakeholders depending on the requirements and clarifications where necessary. The final presentation was made with all the relevant stakeholders via a virtual meeting and the NSP was finalized. Finalized NSP has circulated again among all stakeholders to ensure no further editing was necessary before printing. Later final draft was reviewed by the international reviewers in the WHO SEARO office.

Several guiding principles including high-level political commitment, adopting a human right approach, gender and cultural sensitivity, protecting and promoting equity and social justice and health system strengthening were considered in developing the plan. The document was developed as the first NSP in the WHO SEARO region.

\section{RESULTS AND DISCUSSION}

The participation of at least two meetings from each sector was observed and inputs from all sectors were almost 95\%. This NSP has defined its vision, mission, goal, seven strategic objectives, several strategic directions and major activities under each strategic direction. The vision is a country with a low incidence of cervical cancer and high survival rates with good quality of life and minimal disabilities and suffering from effect of cervical cancer. The goal is set to reach interim targets of cervical cancer elimination in Sri Lanka 2021-2030. Seven strategic objectives were identified for achieving the goal.

\section{Strategic objective 1 - Strengthen primary prevention through HPV vaccination to achieve $\mathbf{9 0 \%}$ coverage}

The first strategic objective addresses the primary prevention through HPV vaccination by securing sufficient and affordable HPV vaccines and increasing the quality and coverage. It will ensure the high coverage with the final dose of HPV vaccination for all girls in 10 -11 years age cohort. Existing island-wide school-based vaccination programme in government and private schools will be strengthened in quality manner. Ways to identify the any missed individuals from the school programme by $\mathrm{MOH}$ clinics at primary health care level. Further, communication and social mobilization strategies have been included to increase awareness in the community and stakeholders to create and sustain the demand for HPV vaccination. Innovations for cost-effective, efficient approaches to HPV vaccination have been introduced.

\section{Strategic objective 2 - Improve cervical cancer screening by achieving $70 \%$ of screened women with a high-performance test by 35 years and again by 45 years of age}

The second strategy aims to improve the cervical cancer screening to achieve $70 \%$ coverage with a highperformance test. Reviewing and revising existing guidelines in line with latest global evidence to introduce HPV DNA testing and other newer screening techniques like self-sampling are considered as important points. The NSP strengthens the improvement of knowledge, attitude and behavior change of general public, including high risk populations. Accessibility and availability of services on screening and early detection in all possible primary, secondary and tertiary institutions will be made aware. Cervical cancer early detection services will be expanded to cover high risk populations, especially female sex workers, irrespective of the age group. Introduction of self-collected sampling of HPV DNA testing to the general population has been identified in the NSP. Integrate screening and treatment services into the essential health service package, ensure an affordable supply of high-performance screening tests with good quality, strengthening laboratory capacities as well as adequate and trained healthcare workforce are the other prioritized strategies. Strengthening training programmes for primary healthcare staff, capacity building programmes and filling cadre positions especially public health midwives and cytoscreeners have identified in the NSP immediately.

\section{Strategic objective 3- Ensure $90 \%$ of women identified with cervical disease (precancerous and invasive cancer) receive treatment}

This strategy highlighted and ensured sustainable and 
equitable access to diagnosis, treatment and care facilities for cervical cancers with all treatment modalities. The improvement of diagnostic services, treatment modalities and palliative care have been included to achieve the treatment coverage for precancerous and cancerous lesions.

Cervical cancer management guidelines together with other relevant management guidelines are ensured to implemented in line with global guidance and local algorithm decided by an expert committee. Radiotherapy including brachytherapy for cervical cancer is included in the NSP considering the equal, availability and accessibility in Sri Lanka. Establishment of referral pathways and people-centered linkages throughout the continuum of care have been identified as important strategic directions. It has identified the need to establish clear referral pathways for women with positive results for HPV DNA/ Pap smear and precancerous lesions to ensure timely management. Further, follow-up mechanisms have been reinforced to ensure no leakage along the care continuum preferably through a unique identifier. Public and private partnership for diagnosis and treatment with appropriate referral mechanism should be strengthened. The multidisciplinary review boards for diagnosis, treatment and rehabilitation in each province have been identified for cervical cancer in the NSP.

Expansion of surgical and oncological treatment modalities as well as cervical cancer diagnostics in the National Cancer Care Institute, Centers of excellence and treatment centers have been included. It has identified the importance of access to treatment modalities as per updated treatment guidelines in all Centers of excellence and treatment centers. The need for scaled up plan for external beam radiation therapy and internal beam radiation therapy (brachytherapy) in identified strategic locations is highlighted. The importance of regular training and deployment of human resources to provide radiotherapy is also highlighted.

This strategic objective also ensured the strengthening and integration of palliative care services. It will be achieved by means of introducing the concept of survivorship to healthcare providers, establishing cervical cancer survivor groups and developing linkages with government, NGOs etc. Further, family members, caregivers and general public should be empowered to provide basic palliative care for cervical cancer patients. The proper coordination of them with government and private healthcare providers is also highlighted. In addition to that, importance of optimizing health workforce competencies to deliver quality cancer care throughout the continuum of care and the reduction of stigmatization associated with cervical cancer have been addressed.

\section{Strategic objective 4 - Strengthen health system support for elimination of cervical cancer}

This strategy highlighted the strengthening of health system support for elimination of cervical cancer. It includes the strengthening of the national programme as well as provincial teams. It has addressed planning, coordination, monitoring and evaluation of preventive and control activities at the NCCP, FHB and Epidemiology unit. Further, it has highlighted the importance of monitoring of the strategies through relevant Technical Advisory Committees and National Advisory Committees. Compulsory reporting of confirmed or suspected cases of cervical cancer through a legal provision and through advocacy has been ensured. Importance of the proper collaboration between NCCP, FHB, National STD/AIDS Control Programme and other partners at all levels to achieve interim targets has been highlighted.

Further, access to essential oncology medicines and other cervical cancer diagnostics, treatments, pharmaceuticals and health products has been addressed. Engagement with private sector providers through public-private partnership and promotion of insurance schemes are suggested. Further, it has highlighted the introduction of systems for improving the quality of healthcare and strengthening innovation and digital technologies for health.

\section{Strategic objective 5 - Improve leaderships, advocacy \& communication}

The fifth strategic objective ensured the provision of highest political leadership for prevention and control of cervical cancer to contribute to achievement of SDGs embracing a multisectoral approach. Advocacy programmes have been emphasized to include cervical cancer as an agenda item at the National Health Council, to promote multisectoral national response and to scale up HPV DNA testing. Further, advocacy is recommended to strategic purchasing of screening, diagnostic and treatment equipment and to offer screening test at low cost price in private sector healthcare providers. In addition to that, it has emphasized effective community education \& empowerment that supports and influence adoption of healthy lifestyles across the lifecycle to reduce risk factors among different target groups. It has elaborated the importance of integrating cervical cancer prevention and control interventions in health promoting settings such as schools, youth settings, work places, hospitals, estates and villages. Involvement of people living with cervical cancer, their families and care givers has also been identified as a measure to improve communication.

\section{Strategic objective 6 - Strengthen cervical cancer information systems and surveillance}

Strengthening information systems as well as surveillance to provide accurate and timely data to monitor the progress of cervical cancer control activities has been identified under this strategic objective. Strengthening National Cancer Registry with linkages to all Population-Based Cancer Registry, Hospital Based Cancer Registry, Pathology laboratory-based and other surveillance systems to ensure timely reporting of cervical cancer data is highlighted. 
Monitoring and evaluation should be strengthening throughout programme planning cycles and across health system building blocks to ensure quality health service delivery. Yearly review for the cervical cancer elimination programme has been recommended with mid-term and end of the term full review.

\section{Strategic objective 7 - Promote research and utilization of its findings}

This strategy has addressed the promotion of research and utilization of its findings for the prevention and control of cervical cancer. It has included the identification of research priorities, promotion of a conducive environment for research and translation of research evidence into practice to strengthen preventive services, treatment and care services of cervical cancer.

The seven strategic objectives of the NSP to reach the interim targets of cervical cancer elimination in Sri Lanka, 2021-2030, would ultimately facilitate the achievement of the global target to eliminate cervical cancer as a public health problem at the end of the century (National cancer control programme 2021c) A strong political commitment from the government of Sri Lanka including health and nonhealth sectors with allocation of resources to support investments in health systems will facilitate the process.

\section{Conclusion and recommendations}

The NSP to reach interim targets in elimination of cervical cancer focused on strategic objectives, strategic directions and major activities. It highlighted the strengthening HPV vaccination, improve cervical cancer screening and improve early diagnosis and treatment facilities. Further, strengthening of health system support, improve advocacy and communication, strengthening cervical cancer information system and research promotion are addressed. It is recommended to implement the monitoring and assess the interim targets annually and to take measures overcome the challenges to achieve the 2030 interim targets.

\section{Acknowledgement}

The authors wish to thank all stakeholders contributed to the National Strategic Plan to Reach the Interim Targets of Cervical Cancer Elimination in Sri Lanka 2021 - 2030 and special acknowledgement to World Health Organization local office and office of the SEARO region.

\section{Conflicts of interest}

The authors declare that they have no known competing financial interests or personal relationships that could have appeared to influence the work reported in this paper.

\section{Ethical approval}

Not applicable. The current study analyzed the development of the National Strategic Plan to Reach the Interim Targets of Cervical Cancer Elimination in Sri Lanka $2021-2030$ and other documents of the Ministry Health that is fully publicly available and the study did not involve a specific person or specimen.

Funding: Not applicable.

Author contributions: Upuli Perera (UP), Thanuja Wickramathunga (TW), Kalpanie Wijewardane (KW,) Saddharma Weerakoon (SW) and Janaki Vidanapathirana (JV), planned the study. UP and TW analyzed the documents. SW, KW and JV helped with results writing. UP \& TW wrote the first version of the manuscript. KW, SW and JV assisted in finalizing the manuscript. All authors have read and approved the final version of the manuscript.

\section{REFERENCES}

de Martel C, Plummer M, Vignat J, Franceschi S (2017). Worldwide burden of cancer attributable to HPV by site, country and HPV type. Int J Cancer. 141(4):664-670.

de Silva AHW, Samarawickrema N, Kasturiratne A, Skinner SR, Wickremasinghe AR, Garland SM (2019). Awareness of human papillomavirus, cervical cancer and its prevention among primigravid antenatal clinic attendees in a tertiary care hospital in Sri Lanka: a cross-sectional study. Sexual Health. 16(3):212-217.

Epidemiology Unit (2020). HPV Vaccination Coverage Report 2020, Epidemiology Unit, Ministry of Health, Sri Lanka.

Family Health Bureau (2019). Annual report 2019. Family Health Bureau, Ministry of Health, Sri Lanka.

National Cancer Control Programme (2020). National guidelines on early detection and referral pathways of common cancers in Sri Lanka. Colombo, National Cancer Control Programme, Ministry of Health, Sri Lanka.

National Cancer Control Programme (2021a). Cancer Incidence Data, National Cancer Registry Sri Lanka. Colombo, National Cancer Control Programme, Ministry of Health, Sri Lanka.

National Cancer Control Programme (2021b). National Strategic Plan on Prevention and Control of Cancer in Sri Lanka, 2020-2024. Colombo, National Cancer Control Programme, Ministry of Health, Sri Lanka.

National Cancer Control Programme (2021c). National Strategic Plan to Reach the Interim Targets of Cervical Cancer Elimination in Sri Lanka 2021-2030. Colombo, National Cancer Control Prgramme, Ministry of Health, Sri Lanka.

Perera KCM, Mapitigama N, Abeysena H (2021). The feasibility of new HPV/DNA test as a primary cervical cancer screening method among 35- years- old ever 
married women in Kalutara district; a cross-sectional study. BMC Public Health. 13:21(1):131.

United Nations (2015). Sustainable Development Goals. United Nations. Available from https://sdgs.un.org/goals.

World Health Organization (2020). Global strategy to accelerate the elimination of cervical cancer as a public health problem. Geneva, WHO.

World Health Organization (2021a). Cervical cancer. Geneva, WHO. Available from https://www.who.int/health-topics/cervical-cancer.

World Health Organization (2021b). Regional implementation framework for elimination of cervical cancer as a public health problem: 2021-2030. New Delhi, WHO, Regional Office for South-East Asia. 